\title{
The effect of a tilted magnetic field on the equilibrium of a pure electron plasma
}

Grant W. Hart

grantwhart@gmail.com

Follow this and additional works at: https://scholarsarchive.byu.edu/facpub

Part of the Astrophysics and Astronomy Commons, and the Physics Commons

\section{Original Publication Citation}

Hart, Grant W."The effect of a tilted magnetic field on the equilibrium of a pure electron plasma." Physics of Fluids B: Plasma Physics 3 (1991): 2987-2993.

\section{BYU ScholarsArchive Citation}

Hart, Grant W., "The effect of a tilted magnetic field on the equilibrium of a pure electron plasma" (1991). Faculty Publications. 719.

https://scholarsarchive.byu.edu/facpub/719 


\title{
The effect of a tilted magnetic field on the equilibrium of a pure electron plasma
}

\author{
Grant W. Hart \\ Department of Physics and Astronomy, Brigham Young University, Provo, Utah 84602
}

(Received 12 March 1991; accepted 23 July 1991)

\begin{abstract}
If the magnetic field in a pure electron plasma containment device is not aligned with the axis of the conducting walls, the electrons in the device will accumulate at the ends of the plasma where the magnetic field lines come closest to the walls and the electrons bound to the field lines can be closest to their image charges. If the plasma is also offset radially from the center (as with an $l=1$ diocotron mode), then more density will accumulate at one end than the other. As the plasma revolves around the center, the electrons will slosh from one end to the other, creating a measurable signal. This signal has been experimentally measured and its origin verified using a three-dimensional equilibrium code. This signal can be used experimentally to align the magnetic field with the conducting walls.
\end{abstract}

\section{INTRODUCTION}

A cylindrical pure electron plasma trap consists of a conducting cylinder that is long compared to its radius (typically $L / r \sim 15$ ), with a magnetic field applied parallel to its axis. The magnetic field confines the electrons in the radial direction and large negative potentials at the ends of the cylinder confine the electrons axially. It has a long been known that aligning the magnetic field with the conducting walls in such a trap is very important to minimize the crossfield transport of the electrons. ${ }^{1}$ It is also known that a tilted field can launch $l=1, k_{z} \neq 0$ diocotron waves. ${ }^{2}$ What has not been investigated, however, is how the tilted field affects the equilibrium and dynamics of the plasma itself. This is not a simple thing to do analytically, because a tilted magnetic field invalidates two of the assumptions usually made in the theory of pure electron plasmas, i.e., an infinite-length plasma and cylindrical symmetry. A tilted magnetic field necessarily implies a finite-length plasma because all magnetic field lines eventually run into a wall; it also destroys the cylindrical symmetry of the problem.

A simple physical picture of what occurs with a tilted field is that the density will tend to accumulate wherever it can be closer to its image charge in the wall, since that is a point of lower energy. Since the plasma is strongly magnetized (the gyroradius for a $1 \mathrm{eV}$ electron in such a trap with a magnetic field of $700 \mathrm{G}$ is $34 \mu \mathrm{m}$, compared to a plasma radius of several centimeters), the electrons are only free to adjust their position along the field lines. This means that the density will tend to accumulate at the ends of the plasma where the field lines bring the electrons closer to the wall. If the plasma is also offset from the center of the trap in the radial direction by a distance $D$ (as is the case for the $l=1$ diocotron mode), then one end of the plasma will be closer to the wall than the other, causing more charge to accumulate at that end. As the $l=1$ diocotron mode causes the plasma to rotate around the center in the azimuthal direction, the two ends will switch roles; the previously closer end becoming the farther end and vice versa. This means the density perturbation will also change ends, causing the perturbed density to slosh back and forth in the system at the diocotron frequency. Since the diocotron frequency is much less than the plasma frequency $\left(f_{d} \sim 25 \mathrm{kHz} \ll f_{p} \sim 15 \mathrm{MHz}\right)$, the perturbation can be viewed as a quasistatic change in the equilibrium rather than as a driven oscillation.

The experimental consequences of this model will be discussed in Sec. II, a threc-dimensional (3-D) equilibrium calculation to verify the signals seen will be discussed in Sec. III, and the conclusions will be presented in Sec. IV.

\section{EXPERIMENTAL MEASUREMENTS}

The experimental apparatus is a cylindrical, pure electron plasma trap of the type used by Malmberg et al. at the University of California at San Diego. ${ }^{1}$ It operates in the usual fill-manipulate-dump cycle: The electrons are allowed into the trap at one end, they are manipulated according to the experimental plan, and then they are dumped out the other end. The basic geometry of this type of trap is a cylindrical conducting wall with a magnetic field applied parallel to the axis of the cylinder. The electrons are confined radially by the magnetic field and axially by negative potentials applied to the conducting wall at the ends of the plasma. The wall is divided axially into rings for making measurements using the image charge of the plasma and for manipulating the length of the plasma. Two of the rings are further subdivided into four azimuthal sectors for measurement and manipulation. The arrangement of the rings in the experiment is shown in Fig. 1. The confining potentials of $-150 \mathrm{~V}$ are applied to the two $2.5 \mathrm{~cm}$ length rings located at the ends of the cylinder. The inner radius of all the rings is $4.0 \mathrm{~cm}$ and the total distance between the inner edges of the confining potential rings is $60 \mathrm{~cm}$, which will roughly be the same length as the plasma. The magnetic field of approximately $700 \mathrm{G}$ is applied along the $z$ direction, the axis of the cylinder. A uniform perpendicular magnetic field of order 1-2 G can also be applied along the entire length of the trap.

The way the rings and sectors of the wall are connected together determines their sensitivity to different types of perturbations when they are used as image charge detectors. 


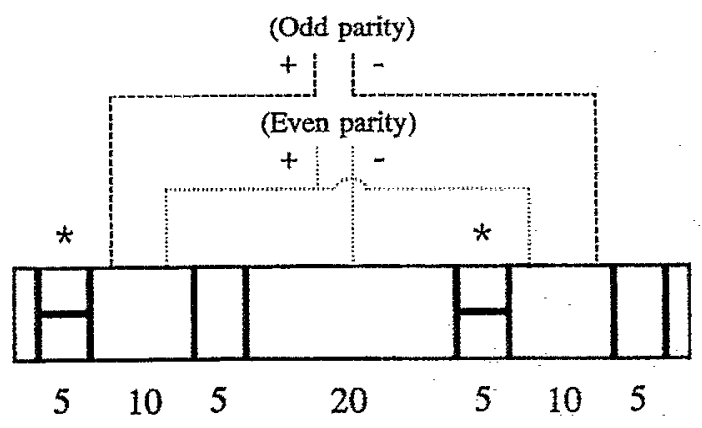

FIG. 1. Positioning of the rings that make up the conducting wall of the pure electron plasma experiment. The rings marked with an asterisk are further segmented into four azimuthal sectors. The length of each ring in centimeters is indicated below it. The confinement potentials are applied to the unmarked short rings at the ends. The dashed lines indicate the connections for an odd parity $l=0$ detector. The dotted lines indicate the connections for an even parity $l=0$ detector.

The detectors can be characterized by their sensitivity to perturbations of the form $e^{i t \theta}$, where $\theta$ is the azimuthal angle. If a sectored ring is connected together as shown in Fig. $2(a)$, it is sensitive primarily to $l=1$ perturbations. The + in the figure corresponds to the positive side of a differential amplifier and the -corresponds to the negative side. It also has some sensitivity to $l=3,5, \ldots$, perturbations. If the ring is connected as shown in Fig. 2(b), it is primarily sensitive to $l=2$ perturbations with some sensitivity to $l=6,10, \ldots$, perturbations.

The image charge on an unsectored ring is due mainly to the charge located directly inside its circumference, with some sensitivity to charge that is located up to about a ring radius away in the axial direction. If the plasma is long and uniform in $z$, then the image charge per unit length will equal the plasma charge per unit length. If the plasma charge density is peaked in $z$, then the wall charge density will be somewhat less strongly peaked in $z$. Because an $l=0$ perturbation has the effect of changing the charge per unit length of the plasma, unsectored rings are termed $l=0$ detectors. To detect the axial variation in the longitudinal line density of the plasma, the two $10 \mathrm{~cm}$ long rings are connected to the two

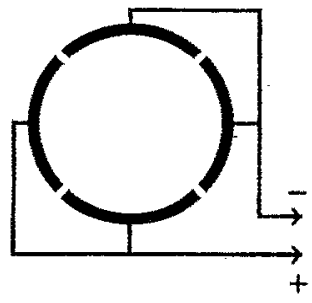

(a)

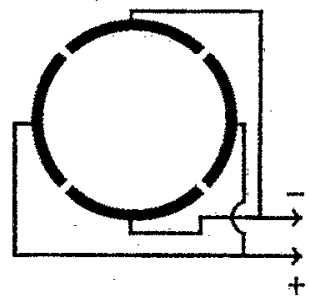

(b)
FIG. 2. The connections of a sectored ring to conflgure it as an $l=1$ or $l=2$ detector. (a) shows the connections for an $l=1$ detector and (b) shows the same for an $l=2$ detector. The + and - on the figure indicate connections to the + and - sides of a differential amplifier, respectively.

sides of a differential amplifier as in Fig. 1. This will detect perturbations that have an odd parity in $z$. To detect an even parity signal, the two $10 \mathrm{~cm}$ rings are connected to the positive side of the differential amplifier and the central $20 \mathrm{~cm}$ ring to the negative side of the same amplifier as also shown in Fig. 1. The two sectored rings (centered at $z=12.5$ and $-27.5 \mathrm{~cm}$ ) are configured as $l=1$ and $l=2$ detectors, respectively.

Because the infut impedance of the differential amplifiers used is quite high (of order $1 M \Omega$ ), the measured output voltage is due to the image charge induced by the plasma distributed over the capacitance to ground of the detector rings and the coaxial cables connecting the rings to the amplifier.

The signal observed on the $l=1$ and $l=2$ detectors is due to the image charge in a section of wall that is short compared to the length of the plasma. Because the detector is short and the tilt angle is small, a good approximation is that the image charge is the same as for an infinitely long rod of charge located at the average radial position of the plasma and having the same average charge per unit length. Because the axial bounce frequency of the electrons is much higher than the diocotron frequency $\left(f_{b} / f_{d} \sim 15\right)$ and the tilt angle is small, the $l=1, k_{z}=0$ diocotron mode can be approximated by having the plasma oriented along the tilted magnetic field and moving as a rigid body in a circle about the field line that goes through the geometric center of the machine (i.e., $r=z=0$ ). The motion of the plasma could be more complex than this since much of the symmetry has been lost, but this assumption is consistent with the data. The radius of the circular motion is $D$. To lowest order, the effect of the sloshing density in the $l=1$ and $l=2$ signals can be ignored.

The geometry ol this model is shown in Fig. 3. The main effect of the tilt on this model is that, at $z \neq 0$, the center of rotation for the plasma (the point marked $r$ in the figure) is no longer at the gecmetric center, but rather is offset from the center of the machine by a distance $\rho$ at an angle $\theta_{p}$, where $\rho$ and $\theta_{p}$ are determined by the assumption that the center of rotation is on the magnetic field line that runs through $r=z=0$. If we think of the tilted magnetic field as consisting of an axiai field plus a perpendicular field, $\theta_{p}$ is the direction of that perpendicular field in the $x-y$ plane.

Previous workers ${ }^{3.4}$ have calculated the image charge expected on a finite size sector of a cylindrical wall due to an

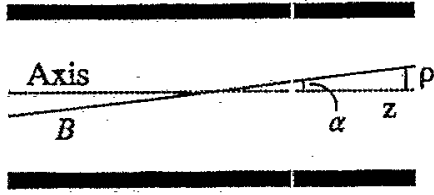

(a)

FIG. 3. The geometry of the tilted magnetic field. (a) shows a side view of the field and $(b)$ shows an end view at an axial position $z$. The perpendicular component of the magnetic field is oriented at an angel $\theta_{p}$ relative to the $x$ axis. The distance $\rho$ is the offset of the magnetic field line that goes through $r=z=0$ from the geometric center of the machine at position $z$. The point labeled $r$ in the figure is the position of the rotation axis for the plasma. 
infinitely long rod of charge. The sector has wall radius $R_{w}$, length $L_{s}$, subtends an azimuthal angle $\Delta \theta$, and has its center located at an angular position $\theta_{s}$ relative to the $x$ axis. The rod of charge has charge per unit length $\lambda_{p}$ and is offset from the center of the cylinder by a distance $R$ at an azimuthal angle $\theta$. Their result for the image charge is

$q_{s}=2 \lambda_{p} L_{s} \sum_{n=1}^{\infty} \frac{1}{n \pi} \sin \left(\frac{n \Delta \theta}{2}\right)\left(\frac{R}{R_{w}}\right)^{n} \cos \left[n\left(\theta-\theta_{s}\right)\right]$.

The relationship between $R, \rho, D, \theta$, and $\theta_{p}$ is illustrated in Fig. 4. The angle $\phi$ in this figure is the angle from the $x$ direction to the rod of charge measured relative to the center of rotation. There is no dependence on the radius of the rod since the electric field outside of an infinitely long axisymmetric rod of charge does not depend on the radial distribution of that charge, even if there are nearby conductors. ${ }^{4}$

We assume that the plasma revolves about the center of rotation in the $-\theta$ direction (the $\mathbf{E} \times \mathbf{B}$ direction for an electron plasma with the magnetic field in the $-z$ direction, i.e., into the paper in the figure) with frequency $\omega$. This implies that $\phi=-\omega t+\phi_{0}$. The relationships that

$$
R \cos \theta=\rho \cos \theta_{p}+D \cos \phi
$$

and

$$
R \sin \theta=\rho \sin \theta_{p}+D \sin \phi
$$

can be used to calculate $R^{n} \cos \left[n\left(\theta-\theta_{s}\right)\right]$. The voltage contributions from each sector (the charge $q_{s}$ divided by the capacitance to ground) can then be added with the appropriate sign determined by the connection of that sector to the differential amplifier as shown in Fig. 2. We take the lowest surviving term of each sum and ignore any part that is constant in time. We then adjust $\phi_{0}$ so that we get a sine term with no phase shift for the $l=1$ detector. This gives us signals of the form

$$
V_{1}=V_{A}\left(D / R_{w}\right) \sin (\omega t),
$$

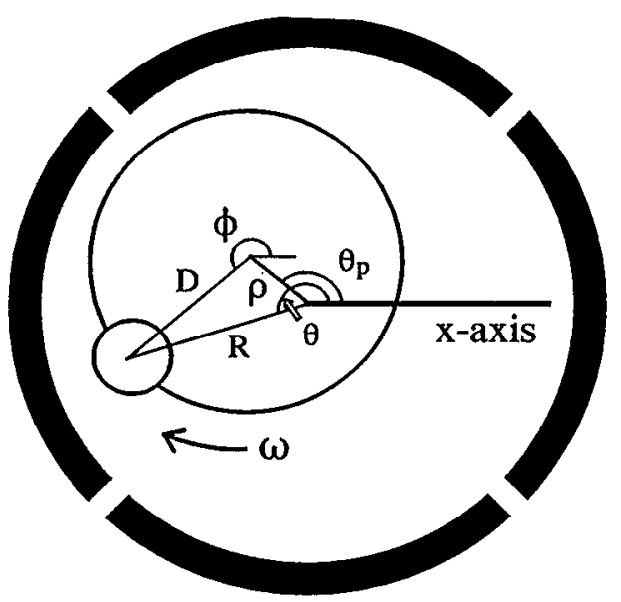

FIG. 4. The relationship between $R, \rho, D, \phi, \theta$, and $\theta_{\rho}$. The plasma revolves with angular speed $\omega$ in the direction indicated around the circle of radius $D$. The size of $\rho$ relative to $R$ and $D$ has been greatly exaggerated for clarity.

$$
\begin{aligned}
V_{2}= & V_{A}\left[2 \frac{D}{R_{w}} \frac{\rho}{R_{w}} \sin \left(\omega t-\theta_{\rho}+\frac{3 \pi}{4}\right)\right. \\
& \left.+\left(\frac{D}{R_{w}}\right)^{2} \sin (2 \omega t)\right],
\end{aligned}
$$

where $V_{1}$ and $V_{2}$ are the voltages on the $l=1$ and $l=2$ detectors, respectively. Here, $V_{A}$ is a constant that depends on $\lambda_{p}, L_{s}$, and the capacitance to ground. The higher-order terms that are neglected in Eq. (4) are at least cubic in products of $D / R_{w}$ and $\rho / R_{w}$. Using numbers typical for the experiment (see later in this section), the next term is a factor of $5 \times 10^{-3}$ smaller. Because of the symmetry of the $l=2$ configuration, there are no higher-order terms in Eq. (5) until sixth-order in products of $D / R_{w}$ and $\rho / R_{w}$. These can truly be considered small. Because $\rho$ is a linear function of $z$ with $\rho=0$ at $z=0$, the $\omega t$ term in Eq. (5) will be a function of $z$, while the $2 \omega t$ term in Eq. (5) and the $\omega t$ term in Eq. (4) will be independent of $z$.

The voltage on the $l=0$ detectors should have the form

$$
V_{0}=V_{B} \sin \left(\omega t+\theta_{p}-\pi / 4\right) \text {, }
$$

because the density perturbation should have a maximum at the time when the plasma is closest to the wall. This occurs when $\phi=\theta=\theta_{p}$. Applying this and the phase relationship between $\phi$ and $\omega t$ used in Eqs. (4) and (5), we get Eq. (6). From the physical model, we can conclude that the constant $V_{B}$ should be some function of $D$, the diocotron radius, since a tilted plasma radially centered in the tube should have no difference in charge density between the two ends, but one that is radially offset should have such a difference. For simplicity, a linear dependence will be assumed. For similar reasons, it will be assumed that $V_{B}$ has a linear dependence on the tilt angle. Both the 3-D equilibrium calculation and the data confirm this linear dependence for small tilts and small $D / R_{w}$.

The general form of the experimental signals on the $l=1, l=2$, and $l=0$ detectors would be (assuming that only the terms shown above are present)

$$
\begin{aligned}
& V_{1}=V_{11} \sin (\omega t), \\
& V_{2}=V_{21} \sin \left(\omega t+\phi_{21}\right)+V_{22} \sin \left(2 \omega t+\phi_{22}\right), \\
& V_{0}=V_{01} \sin \left(\omega t+\phi_{01}\right),
\end{aligned}
$$

if we reference our time relative to $V_{1}$. The first of the double subscripts labels the $l$ value of the detector and the second labels the multiplier of $\omega t$ associated with that quantity.

Experimental data demonstrating these relationships are shown in Fig. 5. The $l=1$ and $l=0$ signals are shown on the upper axes, while the $l=2$ signal and its Fourier decomposition are shown on the lower axes. Here, $\phi_{01}$ is $-45^{\circ}$, $\phi_{21}=135^{\circ}$, and $\phi_{22}=0^{\circ}$, and $V_{21}$ is the amplitude of the dotted curve of lower frequency on the lower axis, while $V_{22}$ is the amplitude of the higher-frequency dotted curve.

Comparing Eqs. (7)-(9) with Eqs. (4)-(6), we see that we have redundant information, allowing us to make sure that the data are consistent with this picture. We can see that $\phi_{22}=0$ and that we have two equations with which to derive $\theta_{p}$ from the experimentally measured phase shifts,

$$
\theta_{p}=\phi_{01}+\pi / 4=3 \pi / 4-\phi_{21}
$$




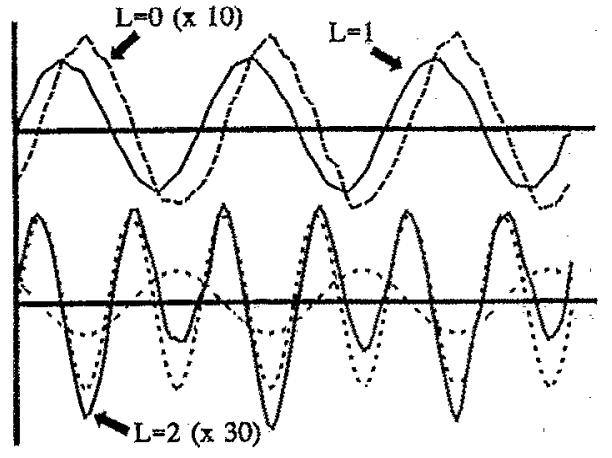

FIG. 5. Data traces showing the amplitude and phase relationships between the $l=1, l=0$, and $l=2$ signais. The solid curve on the upper axis is the $l=1$ signal and the dashed curve is the $l=0$ signal. The solid curve on the lower axis is the $l=2$ signal and the dotted lines indicate the Fourier components of the $l=2$ signal. The signals have been digitally filtered to remove any noise components higher than ten times the fundamental frequency. The amplitude of the $l=1$ signal is approximately $5 \mathrm{mV}$.

which tells us that

$$
\phi_{01}+\phi_{21}=\pi / 2
$$

We can also see that

$$
\begin{aligned}
& D / R_{w}=V_{22} / V_{11}, \\
& \rho / R_{w}=V_{21} / 2 V_{11} .
\end{aligned}
$$

In the small tilt angle approximation,

$$
\alpha=\rho / z_{d}=B_{1} / B
$$

where $\alpha$ is the angle of tilt for the magnetic field, $z_{d}$ is the $z$ position of the $l=2$ detector, and $B_{1}$ is the magnitude of the perpendicular magnetic field. From our previous considerations of the dependence of $V_{B}$ on $D$ and $\alpha$, we would expect that

$$
\alpha \propto \frac{V_{B}}{D / R_{w}} \propto \frac{V_{01}}{V_{11}}
$$

which, within a multiplicative constant, also gives us the magnitude of the perturbation $B_{1}$.

Since $\theta_{p}$ is the direction of the perpendicular field, we can get a measure of both the magnitude and direction of the misalignment between the geometric axis of the machine and the magnetic field from this measurement. Either the $l=2$ or $l=0$ signal combined with the $l=1$ signal is sufficient to make this measurement; and both give the same results. Some care must be used if either the $l=1$ or $l=2$ detector is located at the end of the plasma. The effective $\lambda_{p}$ will be different because the plasma end is near. This changes $V_{A}$ in either Eq. (4) or (5). The exact amount depends on the plasma density and the size of the confinement potential. This effect can be compensated for, however, by measuring the $l=1$ signal on both detectors (one near the center and one near the end) and finding the appropriate factor to multiply the signals on the end detector.

Applying Eqs. (12) and (13) to the experiment with no applied perpendicular magnetic field, the $l=1$ and $l=2 \mathrm{sig}$ nals gave $D=5.0 \mathrm{~mm}$ and $\rho=0.68 \mathrm{~mm}$ at $z=27.5 \mathrm{~cm}$. This implies that $\alpha=2.5 \times 10^{-3} \mathrm{rad}$. Both the $l=2$ and $l=0$ signals gave a perpendicular field direction of $178^{\circ}$ relative to the horizontal. The absolute magnitude of the $l=0$ signal will be discussed in the next section.

A perpendicular field applied to the experiment will change the direction and magnitude of the net perturbation. The resultant perturbation will be the vector sum of the initial perpendicular field plus the applied perpendicular field. Data taken at different applied fields can be fit to this model. Three parameters are allowed to be free in performing this fit, the magnitude and direction of the initial field, and the direction of the applied field. Allowing the parameters to be free enables them to be determined with more accuracy than by a single measurement. It also allows discovery of systematic errors, such as a rotation of the coordinate system, since all angles are measured relative to the orientation of the $l=1$ and $l=2$ detectors, which are invisible inside the vacuum system. It is of course necessary that the parameters derived from this fit agree with other measurements. Figure 6 shows data fit in this marner for two cases, one with the external field coil aligned with the initial perturbation and one with the coil $120^{\circ}$ away. The horizontal axis on the plots is the current in the correction coil. The vertical axis on the top curve is the direction of the net perpendicular field (initial plus applied) and the vertical axis on the lower curve is the relative magnitude of the perpendicular field derived from

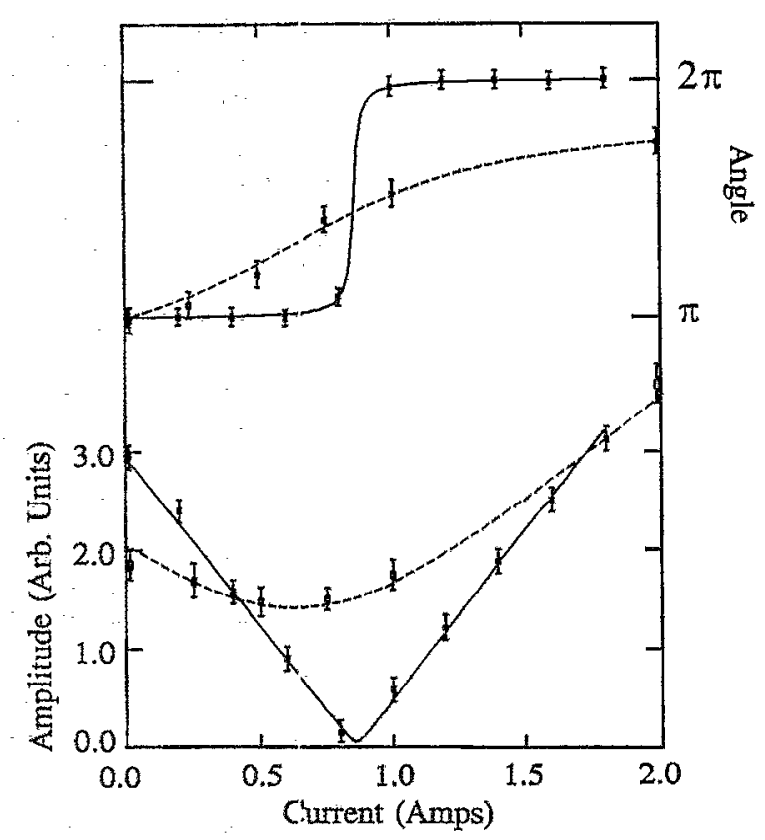

FIG. 6. The magnitude and phase of the $l=0$ and $l=2$ signals as a function of the current in the applied perpendicular magnetic field coil for two different coil orientations. The curves are nonlinear least squares fits of the data to the vector sum of a static field plus the applied field. Three parameters are allowed to be free in the fit, the magnitude and direction of the static field and the direction of the applied field. The top curves show the direction of the perturbation and the lower curves show the relative magnitude of the perturbation. The solid curves (with data points shown as solid circles) are $l=0$ data taken with tire coil aligned with the initial perturbation. The dashed curves with open circle points are $l=2$ data taken with the coil at an angle of $120^{\circ}$ relative to tire initial perturbation. The error bars shown are $2 \sigma$ error bars. 
the $l=0$ or $l=2$ signal. The dashed curves are for the case of the nonaligned external coil and they are taken from $l=2$ data. The $l=0$ data for this case fit equally well to the same parameters. The solid curves are for the aligned external coil case and show $l=0$ data. The derived values for the direction and magnitude of the external perturbation agree in the two fits. The derived direction of the external coil also agrees with external measurements. Figure 6 shows that an external coil can cancel the perpendicular field and therefore align the magnetic field with the axis of the machine. This is true whether the cause of the tilt is an external magnetic field (such as the Earth's field) or a mechanical misalignment. In the latter case, the magnitude of the applied perpendicular field must vary with the magnitude of the solenoidal ficld. The minimum in the lower solid curve in Fig. 6 corresponds to a residual tilt of the order of $10^{-5} \mathrm{rad}$. The good fit of both this data and the data taken when the applied field is not aligned with the initial field shows that the underlying model of the vector addition of fields is appropriate and that the $l=0$ signal depends linearly on the tilt angle.

\section{3-D EQUILIBRIUM CALCULATION}

The model proposed here assumes that the frequency of the diocotron mode is much less than the plasma frequency so that the density will respond quasistatically as the plasma rotates around in the tube. An equilibrium calculation is therefore sufficient to determine the response of the density in this approximation. This calculation must necessarily be three dimensional because there is no cylindrical symmetry in the problem.

In a highly magnetized plasma, the motion perpendicular to the magnetic field is given by the $\mathbf{E} \times \mathbf{B}$ drift of the guiding centers. In the direction parallel to the magnetic field, the equilibrium equation for a plasma with pressure and flow is the parallel component of

$$
n m \boldsymbol{\nabla} \cdot \boldsymbol{\nabla} \mathbf{v}=n q \mathbf{E}-\nabla P \text {. }
$$

In the tilted case, a linear analysis of the equilibrium shows that, if the $\mathbf{v} \cdot \nabla \mathbf{v}$ term is not zero, it is very small compared to the other terms. This leaves us with the Boltzmann condition along the magnetic field that

$$
n=n_{0} e^{-q \phi / k T},
$$

where $\phi$ is the electrostatic potential and $T$ is the plasma temperature. We put the Boltzmann condition into Poisson's equation and get

$$
\nabla^{2} \phi=-\left(q / \epsilon_{0}\right) n_{0} e^{-q \phi / k T} .
$$

Equation (17) is applied only along the field, since that is the only direction that the electrons can freely move. True thermal equilibrium ${ }^{5}$ would require that a modified form of Eq. (18) be satisfied throughout the plasma with $n_{0}$ constant everywhere. This would therefore specify both the radial profile of the plasma as well as its $z$ dependence. That condition does not apply to this casc, however, because the perpendicular equilibration time is much longer than the 100 msec duration of the experiment. Under these conditions, the constant $n_{0}$ in Eq. (18) is constant along the field lines, but can vary arbitrarily perpendicular to them. The perpendicular dependence is determined in the calculation by re- quiring that the total charge (integrated along the field) at a given position be equal to the experimentally measured value.

The solution to Eq. (18) under these conditions using the known boundary conditions of the experiment (specifically the confinement potentials applied at the walls) constitutes the solution to the equilibrium problem. Equation (18) can be solved numerically using the simultaneous overrelaxation (SOR) algorithm. ${ }^{6}$ To speed convergence of the solution, Eq. (18) is slightly modified by taking the linear part of $n$ as a function of $\phi$ [i.e., $(\partial n / \partial \phi) \phi$ ] and subtracting the term associated with it from both sides of the equation before finite differencing it. Doing so gives

$$
\left(\nabla^{2}-\frac{n q}{\epsilon_{0}} \frac{q}{k T}\right) \phi=-\frac{n q}{\epsilon_{0}}\left(1+\frac{q \phi}{k T}\right),
$$

where $n$ is given by Eq. (17). This gives a mathematically equivalent equation with very different numerical properties. The solution of Eq. (19) converges much more rapidly and is numerically stable over a wider parameter range than a direct solution of Eq. (18).

The Poisson solver used to solve Eq. (19) is the twodimensional Poisson solver used by Spencer ${ }^{7}$ in recent work, extended in the $z$ direction to make a 3-D Poisson solver with a cylindrical boundary, but using Cartesian coordinates. The density is assumed to be circularly symmetric about the center-of-mass of the plasma in the $x-y$ plane. This assumption is not really correct for plasmas that are offset from the geometrical center of the machine, ${ }^{8}$ but for offsets and plasma radii typical of the data in this paper $\left(D=5 \mathrm{~mm}, r_{p}=2\right.$ $\mathrm{cm}$ ), the quadrupole moment of the distortion of the plasma density is of the order of $1 \%$. This would correspond to an increase of the plasma size in the $\theta$ direction of about $1 \%$ compared to that in the $r$ direction. Since the calculation used a $50 \times 50$ grid in the $x-y$ plane and the plasma radius was about half of the wall radius, this asymmetry corresponds to a difference of about one-quarter of a grid space. This should have an insignificant effect on the total charge integrated over $x$ and $y$, which is the quantity of interest.

Figure 7 shows contours of perturbed density and perturbed potential in a case that is both tilted and shifted. The plot is a cross section in the $x=0$ plane. The shift is $5 \mathrm{~mm}$ $\left(D / R_{w}=0.125\right)$ and the tilt angle is $2.5 \times 10^{-3} \mathrm{rad}$. Both the shift and the tilt are in the positive $y$ direction, which should give the maximum perturbation for both the density and potential. The confinement potentials are applied on the $2.5 \mathrm{~cm}$ rings at the ends of the machine, producing a plasma that is roughly $60 \mathrm{~cm}$ long. The perturbations are calculated relative to an equilibrium that is shifted by $5 \mathrm{~mm}$ and not tilted, so this figure shows the effects of the tilt on the equilibrium. The perturbed density has two parts, one piece due to the rigid motion of the unperturbed density $\xi \cdot \nabla n$, where $\xi$ is the vector corresponding to the rigid tilt motion, and the other due to the attraction of the electrons to the wall. The $\xi \cdot \nabla n$ piece has been subtracted out of the density plot in Fig. 7. For small angles $\alpha$, the $\xi \cdot \nabla n$ piece contributes no extra charge per unit length as a function of $z$, since it just corresponds to a small radial displacement of the density. The other piece, the change in density due to being closer to the wall at one end than the other, is the one that produces a 


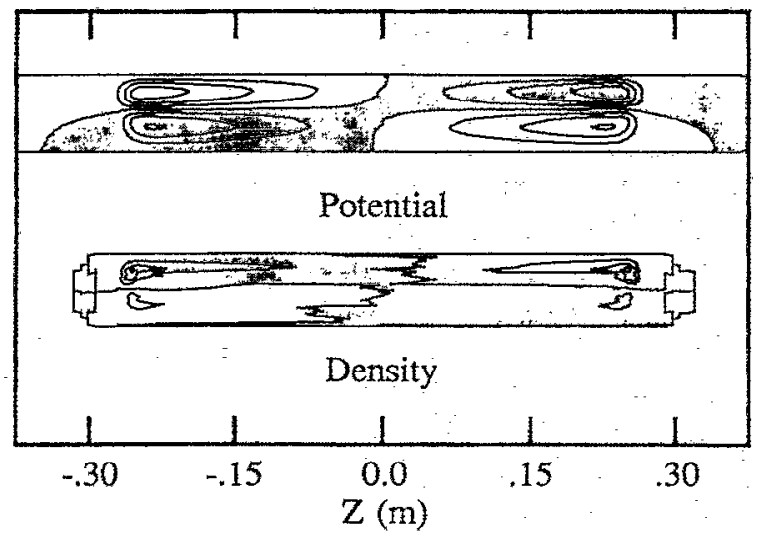

FIG. 7. Contours of perturbed density and perturbed potential for a plasma shifted by $5 \mathrm{~mm}$ and tilted by $2 \times 10^{-3} \mathrm{rad}$, both in the $y$ direction. Thesc plot cross sections in the $x=0$ plane. The perturbation is relative to a plasma shifted by $5 \mathrm{~mm}$ but untilted. The top plot is the perturbed potential and the bottom is the perturbed density. The $\xi \cdot \nabla n$ part of the perturbed density has been subtracted out: The shaded regions in both plots indicate where the perturbation is negative.

change in the charge per unit length. In this case, the two pieces are of roughly equal magnitudes. It can be seen that the density is much more peaked in $z$ than the potential because of the exponential dependence of the density on the potential. The positive density in the upper right-hand quadrant has a much larger magnitude than the negative perturbed density at the same $z$ position, which gives a net positive density at that end. The opposite will be true at the other end, which will have a net negative density.

A Fourier analysis of the perturbed density relative to the tilted axis shows the density perturbation to be mostly $l=1$ in nature. The $l=0$ and $l=2$ contributions are an order of magnitude smaller, and the other contributions are of the same order or smaller than the error in the calculation. The $l=1$ component is typically about $2 \%$ of the peak unperturbed density.

Figure 8 shows the perturbed density integrated over $x$ and $y$ to become the linear charge density as a function of $z$. It also shows the perturbed image charge density in the wall, calculated from the electric field, as a function of $z$. The total perturbed charge induced in the wall (of one sign) agrees with the total perturbed charge in the plasma to within the accuracy of this calculation (a relative error of about $10^{-2}$ in the perturbed quantities). The wall image charge density can be integrated over the positions of the $l=0$ detector rings (shown as dark bars on the axis of the figure) to give the responses of the detectors to this perturbation.

The results of these calculations show that, to within the accuracy of the calculation, the $l=0$ signal is linear in $D / R_{w}$, as expected, up to values of at least 0.125 . The magnitude of the experimentally measured odd parity $l=0$ signal was $1.1( \pm 0.1) \times 10^{-12} \mathrm{C}$, which agrees with the calculated value of $1.17 \times 10^{-12} \mathrm{C}$, within the experimental error. The calculation shows a time-varying, even-parity $l=0$ signal that is more than two orders of magnitude smaller than

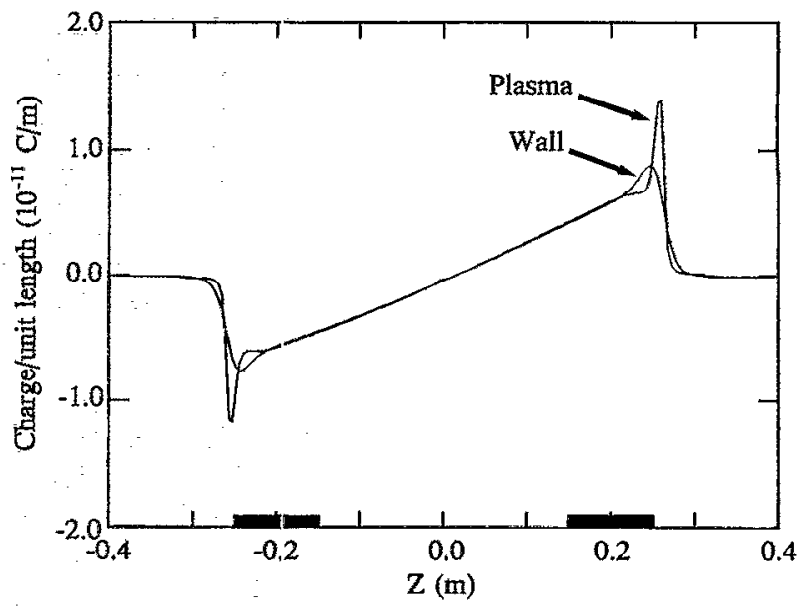

FIG. 8. The perturbed ine charge density of the plasma and the wall as a function of $z$. The bars con the horizontal axis represent the positions of the $l=0$ detector rings.

the odd parity signal, and, indeed, no such signal was observed experimentally.

It is more difficult to calculate the expected $l=1$ and $l=2$ signals, since it is necessary to use a sequence of equilibria as the plasma revolves about the center of the machine. Doing so, however, gives signals that agree (within the accuracy of the calculation) with Eqs. (4) and (5). The calculation also finds a sinall $3 \omega$ signal on the $l=2$ detector and a $2 \omega$ signal on the $l=1$ detector. These unexpected signals are due to the fact thet the sloshing density shows up in the coefficient $V_{A}$ in Eqys. (4) and (5). These signals are about two orders of magnitude smaller than the other signals on the detectors, which makes them similar in magnitude to the first neglected term in Eq. (4).

Using a simple model of the diocotron mode as a rigid rod of charge aligned with the magnetic field, moving with the $(1 / Q) \mathbf{F} \times \mathbf{B}$ drift motion, where $\mathbf{F}$ is the total electric force on the rod and $Q$ is the charge of the rod, the expected frequency shift of the diocotron mode due to the tilted field can be estimated. The change in frequency is due to the fact that the axially averaged radial electric fields in the tilted plasma are slightly different than those in an untilted plasma. This calculation shows a frequency increase of about $1 \%$ for the case shown above, which corresponds to slightly higher fields in the tilted plasma. This is several times smaller than the shot-to-shot variation of the experiment, and therefore could not be observed.

\section{CONCLUSIONS}

A tilted magnetic field causes small but measurable changes in the equilibrium and dynamics of a pure electron plasma. Because of the asymmetry between the two ends of the plasma, a small amount of the density sloshes back and 
forth as the plasma revolves azimuthally inside the machine. Because the diocotron mode frequency $(25 \mathrm{kHz})$ is much less than the plasma frequency ( $15 \mathrm{MHz}$ ), the density sloshing can be considered as a quasistatic modification of the equilibrium, rather than as a driven plasma mode. A 3-D equilibrium calculation of the plasma verifies this model. It also predicts a small (1\%) increase in the frequency of the diocotron mode, but this effect was too small to be seen experimentally.

The presence of this $l=0$ signal is useful experimentally, because it allows a single measurement to determine the magnitude and direction of any magnetic ficld misalignment. This allows a straightforward correction of that misalignment.

\section{ACKNOWLEDGMENTS}

I would like to thank R. L. Spencer and S. N. Rasband for many useful discussions and suggestions about this work.

'J. S. deGrassie and J. H. Malmberg, Phys. Rev. Lett. 39, 1077 (1972).

${ }^{2}$ C. F. Driscoll, Phys. Rev. Lett. 64, 645 (1990).

${ }^{3}$ C. A. Kapetanakos and A. W. Trivelpiece, J. Appl. Phys. 42, 4841 (1971).

${ }^{4}$ K. S. Fine, Yh.D. dissertation, University of California, San Diego, 1988.

${ }^{5}$ T. M. O'Neil and C. F. Driscoll, Phys. Fluids 22, 266 (1979); S. A. Prasad and T. M. O'Neil, Phys. Fluids 22, 278 (1979).

${ }^{6}$ W. H. Press, B. P. Flannery, S. A. Teukolsky, and W. T. Vetterling, $\mathrm{Nu-}$ merical Recipes, The Art of Scientific Computing (Cambridge U. P., Cambridge, 1986), p. 655 .

${ }^{7}$ R. L. Spencer, Phys. Fluids B 2, 2306 (1990).

${ }^{8}$ K. S. Fine, C. F. Driscoll, and J. H. Malmberg, Phys. Rev. Lett. 63, 2232 (1989). 